\title{
Lagrangian foliations and Anosov symplectomorphisms on Kähler manifolds
}

\author{
M. J. D. HAMILTON† and D. KOTSCHICK@ $†$ \\ $\dagger$ Institut für Geometrie und Topologie, Universität Stuttgart, Pfaffenwaldring 57, \\ 70569 Stuttgart, Germany \\ (e-mail: mark.hamilton@math.lmu.de) \\ $\ddagger$ Mathematisches Institut, LMU München, Theresienstr. 39, 80333 München, Germany \\ (e-mail:dieter@math.lmu.de)
}

(Received 8 July 2019 and accepted in revised form 24 August 2020)

\begin{abstract}
We investigate parallel Lagrangian foliations on Kähler manifolds. On the one hand, we show that a Kähler metric admitting a parallel Lagrangian foliation must be flat. On the other hand, we give many examples of parallel Lagrangian foliations on closed flat Kähler manifolds which are not tori. These examples arise from Anosov automorphisms preserving a Kähler form.
\end{abstract}

Key words: Lagrangian foliation, flat Kähler manifold, Anosov symplectomorphism 2020 Mathematics subject classification: 32Q15, 37D20, 53B35 (Primary); 53C12, 53C55, 53D12 (Secondary)

\section{Introduction}

Inthis paper we investigate Kähler manifolds which admit a Lagrangian foliation that is parallel with respect to the Levi-Civita connection. Our first theorem says that the presence of a parallel Lagrangian foliation forces the Kähler metric to be flat.

THEOREM 1. If a Kähler metric admits a parallel Lagrangian foliation, then it is flat.

This is a local result not requiring any compactness assumption. The proof uses the fact that the orthogonal complement of a parallel foliation is also parallel, so that the metric is locally a product. Moreover, in the situation at hand both factors are Lagrangian, and the Levi-Civita connection coincides with the Bott connection of the Lagrangian foliations and is therefore flat.

By Theorem 1, there are very few closed Kähler manifolds with parallel Lagrangian foliations, since they are all Bieberbach manifolds. Nevertheless, in the second part of 
this paper we construct many interesting examples arising from Anosov diffeomorphisms which preserve a Kähler form and are therefore symplectomorphisms. We prove the following criterion for the existence of an Anosov symplectomorphism with respect to a Kähler form on a Bieberbach manifold.

THEOREM 2. A closed flat Kähler manifold $M=\mathbb{R}^{2 n} / \Gamma$ admits an Anosov symplectomorphism $\varphi$ with respect to the Kähler form if there is a hyperbolic matrix $H \in G L(2 n, \mathbb{Z})$ such that $H^{*}\left(\omega_{0}\right)=\omega_{0}$ for the standard Kähler form $\omega_{0}$ on $\mathbb{R}^{2 n}$, and $H$ commutes with the image of the holonomy representation $\rho: \Gamma \longrightarrow G L(2 n, \mathbb{Z})$ of $M$.

In this situation, the stable and unstable foliations for $\varphi$ are parallel and Lagrangian.

The proof is an adaptation of arguments of Epstein and Shub [7] and Porteous [14], who considered the existence of expanding, respectively Anosov, diffeomorphisms, without involving a symplectic or Kähler form. We apply this criterion to show that the holonomy representation of any Bieberbach manifold can be doubled to obtain a flat Kähler manifold which admits an Anosov symplectomorphism with respect to the Kähler form. This doubling construction was first used by Porteous [14] to construct Anosov diffeomorphisms, and later by Johnson and Rees [12] to construct flat Kähler manifolds. It turns out that the structures are compatible, so that doubling produces Anosov symplectomorphisms on flat Kähler manifolds. The existence of parallel Lagrangian foliations on closed Kähler manifolds that are not tori contradicts a claim made by Vaisman in [15] (cf. top of p. 561).

While our motivation for proving Theorem 2 was to find examples of Kähler manifolds with parallel Lagrangian foliations, there is a different, dynamical point of view, which leads one to consider the same class of examples. It is a theorem of Benoist and Labourie [2] that any symplectic manifold admitting an Anosov symplectomorphism with smooth stable and unstable foliations is an infranilmanifold, so that the Anosov symplectomorphism is conjugate to a linear automorphism. Since the only nilmanifolds admitting Kähler structures are tori, the only infranil Kähler manifolds are infra-abelian, i.e. Bieberbach. Theorem 2 produces many examples of this situation. Note that no version of Theorem 1 can be deduced from the theorem of Benoist and Labourie [2], since we do not assume that the Lagrangian foliation in Theorem 1 is induced by an Anosov symplectomorphism.

Initially, our interest in parallel Lagrangian foliations arose from the fact that, together with their orthogonal complements, they give rise to pairs of complementary Lagrangian foliations on symplectic manifolds. Such structures, known variously as bi-Lagrangian [8], para-Kähler [4], or Künneth structures [9], have a very rich geometry. Nevertheless, in this paper we do not use any of the techniques specific to this bi-Lagrangian geometry but work in the framework of standard Kähler geometry.

\section{Flatness of Kähler manifolds with parallel Lagrangian foliations}

Suppose that a smooth manifold $M$ is endowed with a Kähler structure $(J, g)$, meaning that $J$ is an integrable complex structure and $g$ is a positive definite Riemannian metric such that

$$
g(J X, J Y)=g(X, Y) \text { for all } X, Y \in T M,
$$


and the 2-form $\omega$ defined by

$$
\omega(X, Y)=g(J X, Y) \text { for all } X, Y \in T M
$$

is closed and hence symplectic. The Levi-Civita connection $\nabla$ of the Kähler metric $g$ then preserves $g$ and commutes with $J$, and hence is compatible with $\omega$.

We are interested in Lagrangian foliations $\mathcal{F}$ on $M$ which are parallel with respect to the Levi-Civita connection, i.e. $\nabla T \mathcal{F} \subset T \mathcal{F}$. We first show that the orthogonal complement to $\mathcal{F}$ is again a parallel Lagrangian foliation. This result is due to Etayo Gordejuela and Santamaría [8, Theorem 2]. Although we will not need this generality here, we prove the result in greater generality than in [8], not just for Kähler manifolds but for arbitrary almost complex manifolds with a Hermitian metric.

Proposition 3. Suppose $\mathcal{F}$ is a Lagrangian foliation on an almost Hermitian manifold $M$, and that the Levi-Civita connection preserves $T \mathcal{F}$. Then $J(T \mathcal{F})$ is a parallel Lagrangian complement to $T \mathcal{F}$ and is integrable to a Lagrangian foliation $\mathcal{G}$.

Proof. We first show that $J(T \mathcal{F})$ is complementary to $T \mathcal{F}$ and Lagrangian. First, the subbundle $J(T \mathcal{F})$ has the same rank as $T \mathcal{F}$. Second, for $X \in T \mathcal{F}$, we have

$$
g(J X, Y)=\omega(X, Y)=0 \quad \text { for all } Y \in T \mathcal{F}
$$

and hence $J(T \mathcal{F}) \subset(T \mathcal{F})^{\perp}$, where $(T \mathcal{F})^{\perp}$ denotes the $g$-orthogonal complement of $T \mathcal{F}$. Since $g$ is positive definite and $T \mathcal{F}$ has rank half that of $T M$, this shows that $J(T \mathcal{F})=$ $(T \mathcal{F})^{\perp}$ and $J(T \mathcal{F})$ is complementary to $T \mathcal{F}$. It also follows that $J(T \mathcal{F})$ is Lagrangian, because

$$
\omega(J X, J Y)=\omega(X, Y)=0
$$

for all $X, Y \in T \mathcal{F}$.

Since $T \mathcal{F}$ is parallel for the Levi-Civita connection, it follows that $J(T \mathcal{F})=(T \mathcal{F})^{\perp}$ is also parallel and hence integrable.

In this situation, the metric $g$ on $M$ is locally a product metric of the metrics on the factors given by the leaves of $\mathcal{F}$ and $\mathcal{G}$. In order to prove flatness, it is enough to prove that the factors are flat.

We can now prove the main result of this section.

Proof of Theorem 1. Let $(J, g, \omega)$ be a Kähler structure on a smooth manifold $M$ of dimension $2 n$ and $\mathcal{F}$ a Lagrangian foliation with $g$-orthogonal Lagrangian foliation $\mathcal{G}$ given by Proposition 3. The tangent bundle of $M$ decomposes as $T M=T \mathcal{F} \oplus T \mathcal{G}$, and so we can split a tangent vector $X \in T M$ accordingly as $X=X_{F}+X_{G}$. In this situation the Levi-Civita connection of $g$ has a special form.

LEMMA 4. In the situation above, the Levi-Civita connection satisfies:

(1) $\nabla_{X} Y=[X, Y]_{G}$ for all $X \in \Gamma(T \mathcal{F}), Y \in \Gamma(T \mathcal{G})$, and

(2) $\omega\left(\nabla_{X} Y, Z\right)=\left(L_{X} i_{Y} \omega\right)(Z)$ for all $X, Y \in \Gamma(T \mathcal{F}), Z \in \Gamma(T \mathcal{G})$. 
Proof. For the first part, let $X$ be a section of $T \mathcal{F}$ and $Y$ a section of $T \mathcal{G}$. Since the Levi-Civita connection is torsion-free and preserves both $T \mathcal{F}$ and $T \mathcal{G}$, we have

$$
\nabla_{X} Y=\left(\nabla_{X} Y\right)_{G}=\left([X, Y]+\nabla_{Y} X\right)_{G}=[X, Y]_{G}
$$

For the second part, let $X$ and $Y$ be sections of $T \mathcal{F}$ and $Z$ a section of $T \mathcal{G}$. Since the Levi-Civita connection is compatible with $\omega$ and $\mathcal{F}$ is Lagrangian, with (1) we obtain

$$
\begin{aligned}
\omega\left(\nabla_{X} Y, Z\right) & =L_{X} \omega(Y, Z)-\omega\left(Y, \nabla_{X} Z\right) \\
& =L_{X} \omega(Y, Z)-\omega\left(Y,[X, Z]_{G}\right) \\
& =L_{X} \omega(Y, Z)-\omega(Y,[X, Z]) \\
& =\left(L_{X} i_{Y} \omega\right)(Z) .
\end{aligned}
$$

Lemma 5. The Levi-Civita connection is flat along the leaves of $\mathcal{F}$, i.e. $R(X, Y)=0$ whenever $X, Y \in T \mathcal{F}$.

Proof. We need to show $R(X, Y) Z=0$ for all $X, Y$, and $Z$ sections of $T \mathcal{F}$.

Let $V$ be a section of $T \mathcal{G}$. Then parts (1) and (2) of Lemma 4 imply

$$
\begin{aligned}
\omega\left(\nabla_{X} \nabla_{Y} Z, V\right) & =L_{X} \omega\left(\nabla_{Y} Z, V\right)-\omega\left(\nabla_{Y} Z, \nabla_{X} V\right) \\
& =L_{X}\left(\left(L_{Y} i_{Z} \omega\right)(V)\right)-\left(L_{Y} i_{Z} \omega\right)([X, V]) \\
& =\left(L_{X} L_{Y} i_{Z} \omega\right)(V) .
\end{aligned}
$$

It follows that

$$
\begin{aligned}
\omega(R(X, Y) Z, V) & =\omega\left(\nabla_{X} \nabla_{Y} Z, V\right)-\omega\left(\nabla_{Y} \nabla_{X} Z, V\right)-\omega\left(\nabla_{[X, Y]} Z, V\right) \\
& =\left(L_{X} L_{Y} i_{Z} \omega-L_{Y} L_{X} i_{Z} \omega-L_{[X, Y]} i_{Z} \omega\right)(V) \\
& =0 .
\end{aligned}
$$

Since $R(X, Y) Z$ is a section of $T \mathcal{F}$ and $\mathcal{F}$ is Lagrangian, it follows that

$$
\omega(R(X, Y) Z, V)=0 \text { for all } V \in T M,
$$

and hence $R(X, Y) Z=0$ by the non-degeneracy of $\omega$.

Lemma 5 also holds with $\mathcal{F}$ replaced by $\mathcal{G}$. Thus our Kähler metric is locally a product metric of flat metrics and is therefore flat. This completes the proof of Theorem 1.

Remark 1. By the Ambrose-Singer theorem, the flatness result of Theorem 1 can be restated as saying that the reduced holonomy group of the Kähler metric $g$ is trivial. This could be proved by investigating the interaction of the different holonomy reductions that appear in the proof. One is the reduction from $S O(2 n)$ to $U(n)$ given by the Kähler condition; the other is the reduction from $S O(2 n)$ to $S O(n) \times S O(n)$ given by the local product structure. Since $J$ is an isometry of $g$ and interchanges the two factors, there is a further reduction from $S O(n) \times S O(n)$ to $S O(n)$. 


\section{Anosov symplectomorphisms on flat Kähler manifolds}

Recall that a diffeomorphism $\varphi: M \rightarrow M$ is called Anosov if there is a continuous splitting of the tangent bundle into invariant subbundles of positive rank $T M=E^{s} \oplus E^{u}$ such that for all $k>0$

$$
\begin{gathered}
\left\|D \varphi^{k}(v)\right\| \leq a \cdot e^{-b k}\|v\| \quad \text { for all } v \in E^{s}, \\
\left\|D \varphi^{k}(v)\right\| \geq a \cdot e^{b k}\|v\| \quad \text { for all } v \in E^{u},
\end{gathered}
$$

for some positive constants $a$ and $b$.

Here, the norms are taken with respect to some arbitrary Riemannian metric. There is the related concept of an expanding diffeomorphism, for which the second inequality holds for all tangent vectors. This is excluded here by the assumption that both $E^{s}$ and $E^{u}$ have positive rank.

The defining property of an Anosov diffeomorphism is sometimes referred to as the existence of an Anosov splitting $T M=E^{s} \oplus E^{u}$ into stable (or contracting) and unstable (or dilating) subbundles $E^{s}$ and $E^{u}$, respectively. This means that $\varphi$ is hyperbolic everywhere. It is easy to see that when an Anosov splitting exists, it is uniquely determined by $\varphi$, as the contracting and dilating subspaces have to be maximal with these properties.

It is known that the subbundles $E^{s}$ and $E^{u}$ are actually tangent to foliations $\mathcal{F}$ and $\mathcal{G}$ with smooth leaves, although the subbundles are only assumed to be continuous. These foliations are called the stable and unstable foliations of $\varphi$. In general, they are not smooth transversely to the leaves, but they are Hölder continuous.

The following lemma is probably well known to some experts.

LeMma 6. Let $(M, \omega)$ be a closed symplectic manifold admitting an Anosov symplectomorphism $\varphi: M \longrightarrow M$. Then $E^{s}$ and $E^{u}$ are Lagrangian with respect to $\omega$.

Proof. Suppose $v, w \in E^{s}$. Then

$$
\omega(v, w)=\left(\varphi^{*} \omega\right)(v, w)=\omega(D \varphi(v), D \varphi(w))=\cdots=\omega\left(D \varphi^{k}(v), D \varphi^{k}(w)\right) .
$$

Using the auxiliary metric $g$, we find that there is a constant $c$ such that

$$
|\omega(v, w)| \leq c \cdot\|\omega\| \cdot\left\|D \varphi^{k}(v)\right\| \cdot\left\|D \varphi^{k}(w)\right\| \leq c \cdot\|\omega\| \cdot a^{2} \cdot e^{-2 b k} \cdot\|v\| \cdot\|w\| .
$$

Letting $k$ go to infinity, the right-hand side becomes arbitrarily small. Therefore, $\omega(v, w)=0$ and $E^{s}$ is $\omega$-isotropic. By the same argument, with $\varphi^{-1}$ replacing $\varphi$, we conclude that $E^{u}$ is also $\omega$-isotropic. As the two subbundles are complementary, they must be equidimensional and Lagrangian.

Let $M$ be a closed flat Kähler manifold with Kähler form $\omega$. Then its universal covering with the lifted Kähler structure is isomorphic to $\mathbb{R}^{2 n} \cong \mathbb{C}^{n}$ with the standard Kähler form $\omega_{0}$. The fundamental group $\pi_{1}(M)=\Gamma$ is a Bieberbach group given by an extension of the form

$$
1 \longrightarrow A \longrightarrow \Gamma \stackrel{p}{\longrightarrow} F \longrightarrow 1,
$$

where $A \cong \mathbb{Z}^{2 n}$ is the maximal subgroup consisting of pure translations, and $F$, the holonomy group of $M$, is finite. The group $\Gamma$ acts on $A$ by conjugation. Since $A$ is 
abelian, this action factors through the projection $p$, giving rise to a representation $\rho: F \longrightarrow G L(A)$ called the holonomy representation of $M$. One can also consider this as a representation of $\Gamma$ factoring through $p$, as in the statement of Theorem 2 in the Introduction.

We can now prove Theorem 2 stated in the Introduction.

Proof of Theorem 2. The first part is a simple adaptation of arguments in Epstein and Shub [7] and Porteous [14]. Since the latter reference is elliptical and refers back to the earlier [7], and our assumptions are a bit different, we spell out the details.

Let $\ell=|F|$ be the order of $F$. Since the group $G L\left(2 n, \mathbb{Z}_{\ell}\right)$ is finite, there is a power $H^{k}$ of $H$ satisfying $H^{k}=I_{2 n}+\ell \tilde{H}$ with $\tilde{H} \in G L(2 n, \mathbb{Z})$.

The short exact sequence (1) gives rise to a five-term exact sequence in group cohomology. We consider this with twisted coefficients in $A$, with respect to the $\Gamma$-action given by conjugation. The induced action of $A$ on itself is trivial, and the action of $F$ is by the holonomy representation $\rho$. A piece of the five-term exact sequence is:

$$
H^{1}(\Gamma ; A) \longrightarrow H^{1}(A ; A)^{F}=\operatorname{Hom}(A, A)^{F} \longrightarrow H^{2}(F ; A) .
$$

The assumption that $H$ commutes with the image of the holonomy representation means that $H \in \operatorname{Hom}(A, A)^{F}$. Since $H^{k}-I_{2 n}=\ell \tilde{H}$, the image of $H^{k}-I_{2 n}$ in $H^{2}(F ; A)$ vanishes, because in the latter group every element is of order (a divisor of) $\ell$. By exactness, $H^{k}-I_{2 n}$ lifts to an element $[\psi] \in H^{1}(\Gamma ; A)$. A representative $\psi$ is a crossed homomorphism with respect to the $\Gamma$-action on $A$, in other words, a map $\psi: \Gamma \longrightarrow A$ satisfying

$$
\psi(x y)=\psi(x) \cdot \rho(p(x)) \psi(y)
$$

for all $x, y \in \Gamma$. Since $[\psi]$ lifts $H^{k}-I_{2 n}$ from $A$ to $\Gamma$, the restriction of $\psi$ to $A$ is $H^{k}-I_{2 n}$.

Now, given $\psi$, we define $f: \Gamma \longrightarrow \Gamma$ by $f(x)=\psi(x) \cdot x$. This is a homomorphism whose restriction to $A$ is $H^{k}$, and which induces the identity on $F$. Therefore, $f$ is injective and gives an isomorphism between $\Gamma$ and the (proper) subgroup $\operatorname{Im}(f) \subset \Gamma$. By Bieberbach's theorems, any such isomorphism between Bieberbach groups is induced by an affine isomorphism $B: \mathbb{R}^{2 n} \longrightarrow \mathbb{R}^{2 n}$, in the sense that for all $p \in \mathbb{R}^{2 n}$ and $x \in \Gamma$ we have

$$
(f(x))(p)=\left(B x B^{-1}\right)(p) .
$$

The linear part of the affine map $B$ is $H^{k}$, because that is the restriction of $f$ to $A$. Now, the assumption $H^{*}\left(\omega_{0}\right)=\omega_{0}$ means that $B$ is a symplectomorphism with respect to $\omega_{0}$. Moreover, this affine symplectomorphism descends to $M=\mathbb{R}^{2 n} / \Gamma$ by construction, and provides the required Anosov symplectomorphism $\varphi$ of $M$.

Finally, for an affine Anosov diffeomorphism, the stable and unstable foliations are linear and therefore parallel with respect to the standard flat connection. They are Lagrangian by Lemma 6. 
Our main application of Theorem 2 is the so-called doubling construction. Let $N$ be a closed flat manifold of dimension $n$ with fundamental group

$$
1 \longrightarrow \mathbb{Z}^{n} \longrightarrow \pi_{1}(N) \stackrel{p}{\longrightarrow} F \longrightarrow 1
$$

and holonomy representation $\rho: F \longrightarrow G L(n, \mathbb{R})$. Then $N \times N$ is a closed flat manifold of dimension $2 n$ with fundamental group

$$
1 \longrightarrow \mathbb{Z}^{2 n} \longrightarrow \pi_{1}(N) \times \pi_{1}(N) \stackrel{p \times p}{\longrightarrow} F \times F \longrightarrow 1
$$

and holonomy representation

$$
\rho \times \rho: F \times F \longrightarrow G L(n, \mathbb{R}) \times G L(n, \mathbb{R}) \subset G L(2 n, \mathbb{R}) .
$$

We denote by $\Gamma$ the subgroup $(p \times p)^{-1}(\Delta(F))$ of $\pi_{1}(N) \times \pi_{1}(N)$, where $\Delta(F) \subset F \times$ $F$ is the diagonal. This is a subgroup of index $|F|$, and the corresponding covering space of $N \times N$ is a flat $2 n$-manifold $M$ whose fundamental group is given by

$$
1 \longrightarrow \mathbb{Z}^{2 n} \longrightarrow \Gamma=\pi_{1}(M) \longrightarrow F \longrightarrow 1,
$$

with holonomy representation

$$
\begin{aligned}
D(\rho): F & \longrightarrow G L(2 n, \mathbb{R}) \\
g & \longmapsto\left(\begin{array}{cc}
\rho(g) & 0 \\
0 & \rho(g)
\end{array}\right) .
\end{aligned}
$$

We call this the double of the holonomy representation $\rho$ of $N$, and call $M$ the double of $N$.

On $\mathbb{R}^{2 n}$, we consider the constant complex structure

$$
J=\left(\begin{array}{cc}
0 & -I_{n} \\
I_{n} & 0
\end{array}\right)
$$

Since this commutes with the image of $D(\rho)$, it descends to the manifold $M$. This complex structure is Kähler, since it is parallel for the flat metric on $M$. The corresponding Kähler form is

$$
\omega_{0}=\sum_{i=1}^{n} d x_{i} \wedge d y_{i},
$$

where the $x_{i}$ and $y_{i}$ are the linear coordinates on the first and second factors of $\mathbb{R}^{2 n}=$ $\mathbb{R}^{n} \times \mathbb{R}^{n}$. Moreover, the matrix

$$
H=\left(\begin{array}{cc}
2 I_{n} & I_{n} \\
I_{n} & I_{n}
\end{array}\right)
$$

is hyperbolic, commutes with the image of $D(\rho)$, and satisfies $H^{*}\left(\omega_{0}\right)=\omega_{0}$. Therefore, Theorem 2 implies the following.

COROLlary 7. For any closed flat manifold $N$ of dimension $n$, the double $M$ is a flat Kähler manifold of real dimension 2n, which admits an Anosov symplectomorphism whose stable and unstable foliations form a pair of complementary parallel Lagrangian foliations. 
Anosov diffeomorphisms and flat Kähler metrics were first constructed on doubles by Porteous [14] and Johnson and Rees [12], respectively. This corollary shows that the two constructions are compatible, and the Anosov diffeomorphisms one constructs preserve the Kähler form of a flat Kähler metric.

Since every finite group is the holonomy group of a closed flat manifold by a classical result of Auslander and Kuranishi, cf. [16, Theorem 3.4.8], we have the following corollary.

COROLLARY 8. Every finite group is the holonomy group of a closed flat Kähler manifold which admits an Anosov symplectomorphism.

If one does not look for Lagrangian foliations induced by Anosov symplectomorphisms, then one does not have to discuss $H$. Instead, one notes that the two factors of the splitting $\mathbb{R}^{2 n}=\mathbb{R}^{n} \times \mathbb{R}^{n}$ are Lagrangian with respect to $\omega_{0}$. Moreover, this splitting is preserved by $D(\rho)$ and so descends to a pair of complementary Lagrangian foliations on $M$. Unlike the stable and unstable foliations of the Anosov symplectomorphism, these Lagrangian foliations have essentially trivial dynamics. Topologically, $M$ may be thought of as a smooth $T^{n}$-bundle over $N$ with finite structure group $F$. In fact, this is a flat bundle in which the leaves of the horizontal foliation are tori, and are copies of the $F$-covering of $N$. The vertical and horizontal foliations of this flat bundle are the Lagrangian foliations induced by the splitting $\mathbb{R}^{2 n}=\mathbb{R}^{n} \times \mathbb{R}^{n}$. This is an instance of the global action-angle coordinates discussed by Duistermaat [6].

\section{Examples in small dimensions}

In this section, we give concrete examples of closed flat Kähler manifolds of small dimension admitting Anosov symplectomorphisms with respect to the Kähler form.

In real dimension two, the only closed flat Kähler manifolds are tori, or elliptic curves. The Kähler form is just an area form, and any area-preserving Anosov diffeomorphism of $T^{2}$, such as $\left(\begin{array}{ll}2 & 1 \\ 1 & 1\end{array}\right)$, provides an example.

In real dimension four, there are eight distinct closed flat Riemannian manifolds, which admit flat Kähler structures, one of them being $T^{4}$. With the potentially stronger assumption that the structure is not just Kähler but projective algebraic, the other such complex surfaces were classified by Bagnera and de Franchis at the beginning of the 20th century. They not only proved that there are seven families up to deformation equivalence, but also showed that all the complex structures are obtained as certain finite quotients of a product of elliptic curves, so that one does not encounter quotients of irreducible complex tori. Through the Kodaira classification of non-algebraic complex surfaces, it became clear much later that there were no additional (non-projective) flat Kähler surfaces. The finite quotients of a product of elliptic curves are now called hyperelliptic or bielliptic surfaces; the Bagnera-de Franchis classification appears, for example, in [1, p. 148]. From the point of view of the associated Bieberbach groups, this classification reappears in [5, Lemma 2.2], where it is proved that the holonomy group of a closed flat Kähler 4 -manifold is one of the groups $\{1\}, \mathbb{Z}_{2}, \mathbb{Z}_{3}, \mathbb{Z}_{4}$, or $\mathbb{Z}_{6}$. Moreover, the corresponding flat manifold is unique in the first and last cases, whereas for $\mathbb{Z}_{2}, \mathbb{Z}_{3}$, and $\mathbb{Z}_{4}$ there are two distinct manifolds for each holonomy group. This leads precisely to the eight 
possibilities given by tori and the seven Bagnera-de Franchis families. In comparing the two classifications, one needs to remember that the groups used to describe the Bagnera-de Franchis families in [1, p. 148] are sometimes larger than the Riemannian holonomy groups. This is because the minimal toroidal covering space of a flat Kähler surface, whose number of sheets is the order of the holonomy group, may not be a product of elliptic curves, although it turns out to be isogenous to one. To obtain this product of elliptic curves one has to pass to a higher-degree covering.

PROPOSITION 9. A closed flat Kähler manifold of real dimension four admits an Anosov symplectomorphism if and only if the holonomy group has order at most two.

In other words, Anosov symplectomorphisms exist for exactly three of the eight cases in the classification. One is the case of tori, and the other two are the manifolds with holonomy group $\mathbb{Z}_{2}$. One of these manifolds with holonomy $\mathbb{Z}_{2}$ is the double of the Klein bottle.

Proof. There is nothing to prove in the case of tori, so we consider only manifolds with non-trivial holonomy group. Without any assumption about Kähler or symplectic structures, Porteous [14, p. 314] proved that a closed flat 4-manifold admitting an Anosov diffeomorphism has holonomy group of order at most two. In the case of order two, we have the following two families of Kähler surfaces. Let $E_{1} \times E_{2}$ be a product of elliptic curves, and $\tau_{1}, \tau_{2} \in E_{2}$ two distinct elements of order two. The map

$$
\begin{aligned}
\iota_{1}: E_{1} \times E_{2} & \longrightarrow E_{1} \times E_{2} \\
(e, f) & \longmapsto\left(-e, f+\tau_{1}\right)
\end{aligned}
$$

is an involution, and the quotient is one of the manifolds with holonomy of order two. The other one is the quotient of $E_{1} \times E_{2}$ by $\mathbb{Z}_{2} \times \mathbb{Z}_{2}$, where the first copy of $\mathbb{Z}_{2}$ is generated by $\iota_{1}$ and the second by

$$
\begin{aligned}
\iota_{2}: E_{1} \times E_{2} & \longrightarrow E_{1} \times E_{2} \\
(e, f) & \longmapsto\left(e+\sigma, f+\tau_{2}\right),
\end{aligned}
$$

with $\sigma \in E_{1}$ of order two. To show that these manifolds admit Anosov symplectomorphisms, it is enough to exhibit an Anosov symplectomorphism on $E_{1} \times E_{2}$ which commutes with $\iota_{1}$ and $\iota_{2}$.

For simplicity, take $E_{1}=E_{2}=E=\mathbb{C} / \mathbb{Z}^{2}$, the square elliptic curve. Then,

$$
H=\left(\begin{array}{llll}
2 & 1 & & \\
1 & 1 & & \\
& & 2 & 1 \\
& & 1 & 1
\end{array}\right)
$$

acts on $\mathbb{C}^{2}=\widetilde{E \times E}$ as an Anosov symplectomorphism and the action descends to $E \times E$. Moreover,

$$
\left(\begin{array}{ll}
2 & 1 \\
1 & 1
\end{array}\right)^{3}=\left(\begin{array}{cc}
13 & 8 \\
8 & 5
\end{array}\right)
$$


fixes all the points of order two in $E$, and so $H^{3}$ commutes with $\iota_{1}$ and $\iota_{2}$. This completes the proof.

Next, we consider flat Kähler manifolds of real dimension six. Via the doubling construction of Corollary 7, the known classification of closed flat 3-manifolds (see [16, § 3.5]) gives 10 examples of closed flat Kähler threefolds admitting Anosov symplectomorphisms. Their holonomy groups are all abelian but not necessarily cyclic, since the Klein 4-group $\mathbb{Z}_{2} \times \mathbb{Z}_{2}$ occurs.

All the closed flat Kähler manifolds of real dimension six were classified by Dekimpe, Halenda, and Szczepanski [5], who found that there were 174 such manifolds up to diffeomorphism. All but one of them have abelian holonomy groups. We will not try to work out here how many of these admit Anosov symplectomorphisms, beyond the 10 examples obtained by doubling. Instead, we consider the unique example with non-abelian holonomy, described first in [5, p. 367], for which the holonomy is the dihedral group of order eight. In [5] it is said that this example is not projective, because it does not appear in the corresponding classification for the projective case due to Lange [13]. However, since this manifold is Kähler with second Betti number equal to two, it must be projective by the Kodaira embedding theorem. After one of us alerted Johnson to this discrepancy, he clarified the construction and properties of this flat manifold with dihedral holonomy; see [11]. Note that by Johnson's much older result [10], every flat Kähler manifold also admits the structure of a smooth complex projective-algebraic variety. (The claim in [13] to the effect that [10] is erroneous is unjustified and possibly due to a misunderstanding.) It has turned out that the discrepancy between $[5,13]$ was due to a mistake in [13], where the proof that dihedral holonomy cannot appear in the projective case was erroneous. In response to a question from the second author, Catanese and Demleitner located the mistake and gave another description of the manifold in question; see [3]. Their description, in the style of Bagnera and de Franchis, exhibits the flat Kähler manifold as a finite quotient of a product of elliptic curves. We can therefore use it to find an Anosov symplectomorphism by the same kind of argument as in the proof of Proposition 9.

PROPOSITION 10. The unique closed flat Kähler threefold with non-abelian holonomy admits an Anosov symplectomorphism.

Proof. As mentioned before, we use the description of the manifold given in [3]. For simplicity, we take the elliptic curves appearing in the construction to be the square $E=$ $\mathbb{C} / \mathbb{Z}^{2}$. Let $A^{\prime}=E \times E \times E$ and $A=A^{\prime} /(1 / 2,1 / 2,0)$. One considers $r$ and $s$ acting on $A^{\prime}$ by

$$
r\left(z_{1}, z_{2}, z_{3}\right)=\left(z_{2},-z_{1}, z_{3}+\frac{1}{4}\right)
$$

and

$$
s\left(z_{1}, z_{2}, z_{3}\right)=\left(z_{2}+\frac{1+i}{2}, z_{1}+\frac{i}{2},-z_{3}\right) .
$$

It was checked in [3] that $r$ and $s$ induce a free action of the dihedral group of order eight on $A$, and that this action contains no pure translations. The quotient is then the sought flat Kähler manifold with dihedral holonomy. 
In order to prove that this manifold admits an Anosov symplectomorphism, it is enough to find an Anosov symplectomorphism on $A^{\prime}$ which fixes $(1 / 2,1 / 2,0)$ and commutes with $r$ and $s$. On $\mathbb{C} \oplus \mathbb{C} \oplus \mathbb{C}$, we consider

$$
H=\left(\begin{array}{llllll}
2 & 1 & & & & \\
1 & 1 & & & & \\
& & 2 & 1 & & \\
& & 1 & 1 & & \\
& & & & 2 & 1 \\
& & & & 1 & 1
\end{array}\right) .
$$

This descends to $A^{\prime}=E \times E \times E$ as an Anosov symplectomorphism. Since

$$
\left(\begin{array}{ll}
2 & 1 \\
1 & 1
\end{array}\right)^{3}=\left(\begin{array}{cc}
13 & 8 \\
8 & 5
\end{array}\right)
$$

fixes $1 / 2$, we conclude that $H^{3}$ descends from $A^{\prime}$ to $A$. Moreover, $\left(\begin{array}{cc}13 & 8 \\ 8 & 5\end{array}\right)$ fixes $1 / 4$ and $i / 2$, and therefore commutes with $r$ and $s$. This shows that $H^{3}$ induces an Anosov symplectomorphism on the quotient of $A$ by the dihedral group generated by $r$ and $s$.

Acknowledgement. The second author would like to thank F. Catanese, A. Demleitner, and, especially, F. Johnson for discussions and correspondence clarifying the construction of the flat Kähler threefold with non-abelian holonomy.

\section{REFERENCES}

[1] W. Barth, C. Peters and A. Van de Ven. Compact Complex Surfaces. Springer, Berlin, 1984.

[2] Y. Benoist and F. Labourie. Sur les difféomorphismes d'Anosov affines à feuilletages stable et instable différentiables. Invent. Math. 111 (1993), 285-308.

[3] F. Catanese and A. Demleitner. Hyperelliptic threefolds with group $D_{4}$, the dihedral group of order 8 . Preprint, 2018, arXiv:1805.01835v1.

[4] V. Cruceanu, P. Fortuny and P. M. Gadea. A survey on paracomplex geometry. Rocky Mountain J. Math. 26 (1996), 83-115.

[5] K. Dekimpe, M. Halenda and A. Szczepanski. Kähler flat manifolds. J. Math. Soc. Japan 61 (2009), 363-377.

[6] J. J. Duistermaat. On global action-angle coordinates. Comm. Pure Appl. Math. 33 (1980), 687-706.

[7] D. Epstein and M. Shub. Expanding endomorphisms of flat manifolds. Topology 7 (1968), 139-141.

[8] F. E. Gordejuela and R. Santamaría. The canonical connection of a bi-Lagrangian manifold. J. Phys. A 34 (2001), 981-987.

[9] M. J. D. Hamilton and D. Kotschick. Künneth Geometry (London Mathematical Society Student Texts). Cambridge University Press, Cambridge (in press).

[10] F. E. A. Johnson. Flat algebraic manifolds. Geometry of Low-Dimensional Manifolds, Vol. 1 (London Mathematical Society Lecture Notes, 150). Cambridge University Press, Cambridge, 1990, pp. 73-91.

[11] F. E. A. Johnson. A flat projective variety with $D_{8}$-holonomy. Tohoku Math. J. 71 (2019), 319-326.

[12] F. E. A. Johnson and E. G. Rees. Kähler groups and rigidity phenomena. Math. Proc. Cambridge Philos. Soc. 109 (1991), 31-44.

[13] H. Lange. Hyperelliptic varieties. Tohoku Math. J. 53 (2001), 491-510.

[14] H. L. Porteous. Anosov diffeomorphisms of flat manifolds. Topology 11 (1972), 307-315.

[15] I. Vaisman. Basics of Lagrangian foliations. Publ. Mat. 33(3) (1989), 559-575.

[16] J. A. Wolf. Spaces of Constant Curvature, 5th edn. Publish or Perish, Houston, TX, 1984. 